Annuaire du Collège de France 2017-2018

\title{
Anthropologie de la nature
}

\section{Philippe Descola}

\section{OpenEdition}

\section{Journals}

Édition électronique

URL : https://journals.openedition.org/annuaire-cdf/15828

DOI : 10.4000/annuaire-cdf.15828

ISBN : 978-2-7226-0572-5

ISSN : 2109-9227

Éditeur

Collège de France

Édition imprimée

Date de publication : 30 décembre 2020

Pagination : 443-469

ISBN : 978-2-7226-0516-9

ISSN : 0069-5580

Référence électronique

Philippe Descola, "Anthropologie de la nature », L'annuaire du Collège de France [En ligne], 118 | 2020,

mis en ligne le 01 avril 2021, consulté le 22 août 2022. URL : http://journals.openedition.org/annuairecdf/15828 ; DOI : https://doi.org/10.4000/annuaire-cdf.15828 


\title{
ANTHROPOLOGIE DE LA NATURE
}

\author{
Philippe DESCOLA \\ Professeur au Collège de France
}

Mots-clés : anthropologie, nature, collectif

La série de cours " La composition des collectifs : formes d'hybridation » est disponible, en audio et vidéo, sur le site internet du Collège de France (https://www.college-defrance.fr/site/philippe-descola/course-2017-2018.htm), ainsi que les séminaires en relation avec le sujet du cours (https://www.college-de-france.fr/site/philippe-descola/ seminar-2017-2018.htm).

\section{ENSEIGNEMENT}

\section{COURS - LA COMPOSITION DES COLLECTIFS : FORMES D'HYBRIDATION}

Le cours fut consacré à un problème dont on avait jusqu'à présent différé l'examen : les modes de composition des collectifs hybrides, c'est-à-dire de ces assemblages d'êtres et de relations qui semblent relever simultanément d'au moins deux régimes ontologiques distincts. Sans revenir en détail sur ce que sont ces régimes, on a rappelé qu'ils correspondent aux filtres au moyen desquels les humains actualisent, de façon différente selon les milieux où ils ont été socialisés, la myriade de qualités, de phénomènes, d'êtres et de relations que leur environnement offre à leur appréhension. On peut appeler cette actualisation une mondiation, à condition de préciser qu'elle n'aboutit pas à une «vision du monde », à une version parmi d'autres d'une réalité transcendante à laquelle on pourrait avoir un accès intégral ; elle produit un monde au sens propre qui chevauche sur ses marges d'autres mondes qui ont été actualisés par d'autres humains dans des circonstances analogues. Et c'est la relative coöncidence de certains de ces mondes, les repères communs et les expériences partagées dont ils témoignent, qui donnent lieu à ce que l'on appelle d'ordinaire « une culture ».

On avait examiné au cours des années précédentes les principaux filtres grâce auxquels cette mondiation s'opère, des filtres conçus comme autant de façons contrastées de détecter des continuités et des discontinuités dans les plis du monde. 
Sous les noms conventionnels qu'on leur a donnés - animisme, totémisme, analogisme, naturalisme -, ces filtres ne constituent pas une typologie empirique, mais un modèle général des systèmes de qualités repérables dans les objets du monde. Ce modèle se décline en combinaisons dont chacune peut s'incarner çà ou là dans une ontologie qui en synthétise de façon ostensible les principes élémentaires : soit une continuité morale entre humains et non-humains, et une discontinuité de leurs dimensions physiques (animisme) ; soit une discontinuité morale et une continuité physique (naturalisme) ; soit une double continuité morale et physique, mais répartie en blocs discontinus d'humains et de non-humains (totémisme) ; soit enfin une double discontinuité, physique et morale, que des réseaux de correspondance s'efforcent en vain de rendre continue (analogisme). De ce point de vue, par exemple, on peut dire que l'animisme est bien représenté par l'ontologie des Indiens d'Amazonie, le totémisme par l'ontologie des Aborigènes australiens, le naturalisme par l'épistémologie néo-kantienne ou l'analogisme par l'ontologie des Indiens de Mésoamérique. Assez souvent, toutefois, ces systèmes de qualités n'existent qu'à l'état de tendance ou se recouvrent partiellement. Plutôt que de les considérer comme des cosmologies closes et compartimentées ou des cultures au sens classique, il faut donc les appréhender comme les conséquences phénoménales de quatre types d'inférence au sujet de l'identité des existants qui nous entourent ou que nous nous plaisons à imaginer. N'importe quel humain est capable de mobiliser l'un ou l'autre de ces types d'inférence selon les circonstances, mais les jugements d'identité récurrents qu'il aura tendance à produire (tel existant appartient à telle catégorie et peut être rangé avec tel ou tel autre existant) suivront la plupart du temps le genre d'inférence privilégié par la communauté au sein de laquelle il a été socialisé.

Lors des années antérieures, on avait présenté les formes de collectif qui correspondent à chacune de ces modalités de mondiation en puisant dans l'ethnographie et l'histoire les exemples les plus conformes au modèle. En Amazonie, dans le nord de l'Amérique du Nord ou en Sibérie, par exemple, les collectifs animistes peuvent être définis comme des tribus-espèces. Ce sont des regroupements d'êtres dont l'intériorité est analogue à celle des humains, ce qui fait d'eux des sujets sociaux de plein droit, mais qui se différencient aussi les uns des autres par paquets du fait de dispositions physiques particulières, chaque tribuespèce ne pouvant avoir accès qu'au segment du monde qu'elle est prédisposée par sa nature à actualiser. Pour le totémisme, que l'on a choisi d'illustrer pour l'essentiel avec l'exemple des aborigènes australiens, chaque collectif correspond à une classe totémique à l'intérieur de laquelle les similitudes entre humains, animaux et plantes sont fondées non pas sur une ressemblance morphologique, mais sur le partage d'un ensemble de qualités physiques et morales que le prototype totémique - généralement désigné par un nom d'animal - transmet génération après génération aux individus humains et non humains composant le groupe qui porte son nom. Mais si ces modèles de collectifs animistes et totémiques sont typiques des institutions amazoniennes ou australiennes, il arrive aussi souvent que les formes sous lesquelles des collectifs se présentent à l'observateur soient moins clairement identifiables parce qu'elles combinent des traits de deux ontologies. Il fallait donc se pencher sur les modalités d'hybridation entre les modèles et c'est à quoi le cours de l'année fut consacré. On a procédé par étude de cas afin d'aborder des modalités différentes d'articulation, c'est-à-dire portant sur des champs de pratiques distincts au sein de chaque collectif, en privilégiant deux formes d'hybridation : entre l'animisme et le totémisme, d'une part, entre l'animisme et l'analogisme d'autre part. La première 
forme de combinaison fut examinée au moyen d'exemples amérindiens : les Bororo du Brésil et les Wayuu de la péninsule de la Guajira, tandis que l'analyse de la seconde a reposé sur des exemples ethnographiques issus d'Asie du Sud-Est.

\section{Animisme et totémisme : la variante bororo}

L'examen de l'articulation entre régime animiste et régime totémique chez les Bororo s'est appuyé sur la remarquable ethnographie de Jon Christopher Crocker, Vital Souls, dans laquelle il propose une opposition entre deux modes d'existence caractérisant à des degrés divers tous les êtres du cosmos de cette population du Brésil central, opposition dont on s'est efforcé de montrer qu'elle renvoie à un contraste entre animisme et totémisme ${ }^{1}$. Les Bororo distinguent en effet au moyen de deux termes vernaculaires, aroe et bope, les domaines de compétence nettement différenciés de deux types de spécialistes rituels : aroe caractérise le monde éternel et immuable des formes logiques, tandis que bope évoque le flux de la réalité quotidienne en perpétuelle mutation. Aroe est une essence nominative dotée d'attributs que Crocker appelle «totémiques ». En effet, chaque modalité d'être, chaque espèce de plante et d'animal, est subsumée sous une paire transcendante qui exprime son essence parfaite et qui est appelée l'aroe de l'espèce. Les aroe sont plus grands et plus beaux que les individus dont ils incarnent le principe, ils constituent un prototype dont les incarnations individuelles n'offrent que des versions amoindries. Chaque clan bororo est associé à une paire d'aroe qui lui donne sa visibilité classificatoire en le définissant par un nom. En outre, il existe au sein de chaque clan une subdivision duelle qui recoupe la séparation des aroe en paires, chaque sous-clan étant lui-même divisé en groupes de noms contrastés par des différences plus spécifiques. L'ensemble de ces subdivisions forme un lexique des essences qui sert à conférer un nom - associé à un ornement - à chaque membre du clan. Les membres d'un clan partagent donc une même essence nominative définissant la personnalité sociale de chaque individu. La composante matérielle de la personne participe, quant à elle, d'un autre principe, dit bope. À l'opposé des aroe qui organisent le monde social et physique en types immuables, les bope sont la cause de tous les changements, le principe de toute transformation. Ils instillent la fertilité, ils commandent les pluies et les désordres atmosphériques, toute mort leur est imputable. Les bope sont à la source du rakare, l'élan vital, lequel existe en quantités finies, chaque individu en étant pourvu une fois pour toutes par ses parents. La copulation et la rupture des interdits font diminuer le stock d'élan vital, entraînant ainsi le vieillissement et la mort.

Une leçon fut consacrée à l'analyse des phénomènes bope. Tel que le décrit Crocker, le monde des bope n'est pas tant qualifiable comme une collection d'essences en perpétuelle transformation, pour reprendre l'une de ses formules, que comme l'expression d'une ontologie très commune dans l'Amérique du Sud des basses terres que l'on peut appeler «l'animisme prédateur». Le principe en est simple : bien des plantes et des animaux, notamment ceux dont se nourrissent les humains, ou bien sont eux-mêmes des esprits, ou se trouvent sous la dépendance d'esprits qui les contrôlent à la manière de pasteurs chargés de veiller sur un troupeau. Ces non-humains sont soit

1. Jon Christopher CROCKer, Vital Souls. Bororo Cosmology, Natural Symbolism, and Shamanism, Tucson, University of Arizona Press, 1985. 
porteurs d'une énergie vitale si puissante qu'elle présente un danger pour ceux qui l'absorbent, soit dotés d'une intériorité analogue à celle des humains qu'il faut pouvoir désactiver afin de consommer sans dommage la chair de ceux qu'elle anime. Les humains doivent ainsi trouver des médiations rituelles pour s'accommoder avec les esprits et s'alimenter des plantes et des animaux sur lesquels ces derniers exercent leur juridiction. Cela peut s'accomplir grâce à l'échange, en faisant des offrandes aux esprits maîtres en contrepartie du droit de manger les animaux et les plantes après avoir chassé les premiers et cultivé ou cueilli les secondes. C'est la voie que suivent les Bororo lorsqu'ils offrent aux esprits bope les prémices de chaque récolte de maïs ou lorsqu'ils leur livrent par l'intermédiaire du chamane bope des morceaux du gibier qu'ils ont rapporté ou des fruits qu'ils ont collectés. Car c'est la mission principale du chamane bope que de rendre aux esprits une partie de ce qu'ils ont concédé aux humains, en s'en alimentant lui-même au profit des bope qu'il abrite, devenant ainsi le vecteur physique d'une transaction dont la finalité principale est d'amadouer les esprits par des offrandes afin qu'ils s'abstiennent de nuire aux humains. De plus, en consommant de la nourriture bope au profit des esprits, le chamane bope réduit le pouvoir nocif de ces aliments pour que les humains puissent les consommer. Le chamane combine ainsi deux opérations : un retour obligatoire dans l'échange - une partie de l'animal ou de la plante soustraits aux bope leur est donnée à manger - et une opération de décontamination, c'est-à-dire, en bonne logique animiste, de désubjectivation ; celle-ci consiste à dépersonnifier la plante ou l'animal pour ne pas avoir à consommer, avec sa chair, son intériorité agissante, source d'effets négatifs sur celui qui s'y risque. Toutefois, au-delà de cet échange entre des personnes humaines et non humaines médiatisé par les chamanes bope, on peut aussi voir cette circulation des flux vitaux entre les plantes, les animaux, les humains et les esprits comme une sorte de prédation généralisée ou de vaste chaîne trophique dans laquelle les humains occupent une position intermédiaire entre ceux qui servent toujours de proie alimentaire et sexuelle et ceux qui ne font que manger les autres, un modèle classique en Amérique du Sud. L'intervention du chamane ne serait alors qu'une tentative de circonvenir ce cycle de la dévoration mutuelle en le rendant moins dangereux pour les humains.

On examina ensuite l'autre versant du contraste entre animisme et totémisme chez les Bororo, le monde des aroe. Les chamanes aroe - ils ont à présent disparu - sont complémentaires des chamanes bope : tandis que les seconds sont les agents passifs de la décontamination des nourritures bope, les premiers sont des spécialistes liturgiques détenteurs d'un savoir sur les connexions entre ces éléments relativement abstraits que sont les aroe, à savoir principalement les ombres des ancêtres, les âmes des humains vivants et toutes les formes d'êtres totémiques associées aux clans bororo, principalement des animaux. Ces essences aroe sont difficiles à appréhender car, à la différence des bope, elles demeurent éloignées de la vie quotidienne et fonctionnent plutôt comme des principes classificatoires au moyen desquels toute chose peut être catégorisée, comparée, différenciée, typologisée, nommée et personnifiée. Leur habitat souterrain est un reflet déformé du monde réel, composé de deux villages où résident les âmes des ancêtres et divisé en huit sections, chacune habitée par les aroe correspondant aux totems de chacun des huit clans bororo. Si le régime animiste et le régime totémique se développent dans deux champs de la pratique nettement distingués, ils manifestent pourtant un intéressant parallélisme dans l'un des aspects centraux de leur expression, à savoir l'usage des animaux au profit des humains. Il existe en effet dans le régime aroe un symétrique des nourritures bope, ce que les Bororo appellent «les animaux de revanche», des 
prédateurs carnivores pour l'essentiel, tués rituellement à la mort d'un humain et qui servent d'aliments à l'aroe du défunt. Tant que l'animal n'a pas été tué, l'aroe du mort est en colère car il ne peut devenir une essence aroe et s'intégrer au système eschatologique bororo. La mise à mort de l'animal de revanche produit une nouvelle essence par la destruction d'un non-humain, lequel est un déclencheur et non une substance car il n'est pas mangé. Il ne s'agit donc pas d'un acte d'incorporation destiné à rétablir un équilibre dans la circulation des flux entre humains et nonhumains comme ce peut être le cas ailleurs en Amazonie en contexte animiste. En outre, une autre fonction importante du chamane aroe est de procurer des animaux bope aux chasseurs et aux pêcheurs, soit en se transformant en gibier ou en poisson afin de rabattre les proies vers ses congénères, soit en se faisant tuer par eux sous un avatar animal. Il soigne enfin les afflictions engendrées chez les humains par des offenses commises contre les aroe, essentiellement des ruptures d'interdits sexuels.

Un premier point de l'opposition entre bope et aroe sur lequel il faut insister est qu'elle est conceptuelle et non essentielle; elle désigne deux régimes d'existence représentés par des êtres, non deux types d'être dont les formes d'existence seraient incommensurables. Tous les êtres du cosmos bororo sont à la fois bope et aroe, leur échelonnement le long d'un continuum étant le produit d'une différence de valence entre ces deux principes; ainsi, les plus bope des bope, les animaux bope, ont néanmoins un principe d'identité aroe, tandis que les animaux aroe, les animaux de revanche et les animaux totémiques aroe sont soumis aux contraintes de la vie organique et possèdent donc une dimension bope. Même si elle prend une forme institutionnelle visible avec l'opposition entre les statuts et les fonctions des deux classes d'officiant rituel, l'opposition entre bope et aroe est un modèle théorique en partie issu de l'imagination structuraliste de Crocker, non un modèle vernaculaire de la division du monde bororo en deux classes d'êtres nettement séparées. C'est un moyen commode de subsumer sous des catégories nommées des modalités distinctes d'existence qui renvoient bien par ailleurs, et de façon explicite pour les Bororo, à deux champs bien différenciés de la vie collective : d'une part, les pratiques et les représentations qui concernent la catégorisation des humains et des non-humains, leur inclusion dans des classes contrastives et la transmission de leurs rôles et de leurs prérogatives à l'intérieur de ces classes; d'autre part, les pratiques et représentations qui concernent les formes d'interaction entre les êtres, notamment les relations alimentaires et sexuelles, la croissance, la senescence, la prédation mutuelle des organismes humains et non humains, et la circulation des flux entre les étages du cosmos. Chacun de ces deux champs différenciés de la vie des humains et des non-humains est structuré par un régime ontologique lui aussi bien différencié : d'une part, un régime animiste dans lequel plantes et animaux se trouvent organisés en autant de collectifs autonomes qu'il y a d'espèces, sous la juridiction des esprits bope, collectifs qui entretiennent un rapport de prédation mutuelle avec les collectifs humains ; d'autre part, un régime totémique, dans lequel les plantes et les animaux, parfois les mêmes que les précédents, deviennent des « essences nominales », pour reprendre l'expression de Crocker, c'est-à-dire un lexique de signes indiciels incarnés tout à la fois dans des êtres en partie invisibles - les âmes des Bororo vivants et de leurs aïeux -, dans des artefacts et des pratiques rituelles, et surtout dans des prototypes de plantes et d'animaux qui viennent donner une identité propre à chacune des subdivisions d'humains et de non-humains qu'ils qualifient.

On n'est donc pas obligé de suivre Crocker lorsqu'il envisage le totémisme des Bororo dans la perspective classificatoire et dénotative qu'il a empruntée à Lévi- 
Strauss, perspective dans laquelle les animaux et les plantes aroe associés à chaque groupe humain ne seraient que des étiquettes utilisant un modèle de pensée fourni par la nature - la discontinuité morphologique et comportementale des espèces afin de conceptualiser la discontinuité sociale entre clans et sous-clans. Car les aroe bororo sont bien parties prenantes de ces segments, et non de simples signes pour les dénoter à la manière d'un langage symbolique ; ils en constituent à la fois le principe de continuité temporelle - les âmes des morts - et la substance concrète, sous la forme de populations différenciées de plantes et d'animaux - les totems claniques. La différence majeure avec le totémisme que l'on pourrait appeler «standard »- le totémisme australien - est l'absence chez les Bororo d'une identité substantielle et comportementale entre les membres humains et non humains d'un clan totémique. Une identité existe bien, mais sa nature précise est malaisée à définir ; Crocker luimême emploie pour la qualifier des expressions paradoxales : « le clan [...] est perçu comme fondé sur des substances communes, mais qui concernent l'identité logique, non la matière physique ${ }^{2} \gg$. En quoi consiste cette identité logique substantive ? Il semble qu'elle soit d'abord spatiale. Dans cet empyrée platonicien qu'est le monde souterrain des aroe, les essences nominales de tous les êtres, visibles et invisibles, y compris de ceux intégrant des collectifs non bororo, sont présentes sous la forme d'aroe dans l'un ou l'autre des huit secteurs correspondant aux huit clans. En portugais, les Bororo disent des aroe que ce sont des «âmes »; ils les définissent aussi parfois comme «l'esprit » d'une espèce de plante ou d'animal. À l'instar des êtres du Rêve australien, il s'agit donc bien de prototypes des existants, subdivisés en classes nommées, et répartis dans des lieux séparés du cosmos. La différence avec l'Australie vient de ce que ces prototypes ne s'incarnent pas physiquement dans les humains et les non-humains de chaque groupe totémique ; ils cohabitent avec les âmes des morts du clan dans le secteur du cosmos souterrain qui leur est assigné et l'on doit régulièrement personnifier certains d'entre eux dans les rituels.

S'il ne s'agit pas d'un mécanisme de genèse différenciée des prototypes d'humains et de non-humains de chaque classe, quels sont les principes qui président à l'association dans un même ensemble des aroe ? Les Bororo sont peu diserts sur la question. L'association entre un prototype aroe et un clan se voit légitimée par le fait qu'un ancêtre du clan, écrit Crocker, «a vu et nommé cette entité ${ }^{3}$ ». Il s'agit donc d'un processus d'objectivation dans lequel, au contraire du totémisme australien, ce n'est pas le prototype totémique qui est à l'origine de l'être qui lui est associé dans la classe totémique, mais c'est un membre de la classe totémique qui actualise en le nommant un être nouveau, lequel devient par cet acte un prototype. Pour autant, le totémisme bororo ne relève pas du totémisme classificatoire à la Lévi-Strauss puisque, tout comme en Australie, l'ensemble des objets du monde bororo - les plantes, les animaux, mais aussi des humains non bororo, des éléments du relief, des parties de l'anatomie humaine, des artefacts, des esprits - est susceptible d'être distribué sous la forme d'essences nominales entre les huit segments totémiques ; on ne peut donc pas parler ici, comme dans Le Totémisme aujourd'hui ${ }^{4}$, d'une

2. Jon Christopher CROCKER, «Selves and Alters among the Eastern Bororo», in D. Maybury-Lewis (dir.), Dialectical Societies: The Gê and Bororo of Central Brazil, Cambridge (Mass.), Harvard University Press, 1979, p. 257.

3. Ibid., p. 265.

4. Claude LÉvi-Strauss, Le Totémisme aujourd'hui, Paris, PUF, 1962. 
transposition des écarts différentiels observables dans la nature aux écarts différentiels construits entre des groupes sociaux, puisque bien des éléments composant les huit ensembles totémiques ne sont pas des espèces qui présenteraient des discontinuités naturelles. Sans être tout à fait homologue au totémisme australien, le totémisme bororo est donc bien un régime ontologique qui distribue dans des classes homogènes nommées des êtres humains et non humains associés pour des motifs a priori : parce qu'ils sont de la même sorte en tant que types logiques, parce qu'ils ont été actualisés par un membre de la classe où ils sont rangés, parce que leurs prototypes résident ensemble dans les mêmes secteurs du monde.

Chez les Bororo, les contrastes entre régime animiste et régime totémique sont fort nets : le premier renvoie aux échanges d'énergie entre des groupes d'humains et de non-humains différenciés par leurs corps - chaque espèce est un collectif qui est prédateur ou proie par rapport aux autres; tandis que le second concerne la transmission d'identités différenciées au sein d'un macro-ensemble d'humains et de non-humains découpés autrement que dans les collectifs animistes, puisque regroupés non plus par espèce de corps, mais par espèce d'âmes, les aroe claniques. Autrement dit, le totémisme bororo peut être vu comme une expansion logique de l'animisme, une expansion dans laquelle, par contraste avec la situation normale dans cette dernière ontologie où les êtres se différencient par leurs physicalités et non par leur intériorité, le totémisme introduit une distinction supplémentaire, entre les âmes ou essences aroe, qui vient amplifier la fragmentation et l'autonomie de chacune des composantes du cosmos. Toutefois, et ainsi qu'il en va également dans le totémisme australien, ce morcellement ontologique est contrebalancé par la dialectique des échanges entre les deux moitiés et entre les deux modes d'existence. En effet, le spécialiste rituel en charge de chacun de ces modes n'acquiert la complétude de son statut que parce qu'il est la condition de l'existence du mode complémentaire du sien. Car c'est bien le chamane aroe qui rend la chasse possible et qui fonde par son action le rôle du chamane bope dans le système animiste, de même que c'est le chamane bope qui, par son rôle de défenseur de l'âme aroe des humains, rend pertinent le travail liturgique du chamane aroe. Le système bororo offre ainsi l'image de deux grilles ontologiques superposées concernant les mêmes êtres et qui sont actualisées alternativement selon les circonstances: dans les rapports de prédation alimentaires et sexuels pour ce qui est de la grille animiste, dans la transmission d'identités et de prérogatives différenciées pour ce qui est de la grille totémique.

\section{Animisme et totémisme : la variante wayuu}

On consacra les leçons suivantes à un autre cas de combinaison entre régime totémique et régime animiste, celui des Wayuu de la Guajira colombienne et vénézuélienne, cas qui se distingue du précédent en ce que la grille totémique s'y rapproche beaucoup plus du modèle australien : une véritable identité substantive et comportementale existe en effet entre les membres humains et non humains des segments totémiques. On a d'abord rappelé le caractère exceptionnel des Wayuu au regard des autres Amérindiens des basses terres tropicales d'Amérique du Sud. Beaucoup plus nombreux que les autres groupes ethniques de la région, enclavés dans une péninsule aride bordée par la mer des Antilles où ils ont mené une existence très autonome pendant plusieurs siècles, ils adoptèrent l'élevage extensif peu de temps après l'arrivée des Espagnols, et le développèrent au point d'en faire l'axe 
central de leur vie sociale, à la manière des sociétés du cattle complex en Afrique de l'Est. L'adoption de l'élevage est d'autant plus intrigante que les Wayuu partagent par ailleurs avec les autres Amérindiens des basses terres une idéologie cynégétique - sinon une pratique intensive de la chasse - fortement marquée par l'animisme et qui confère aux esprits maîtres des animaux une influence considérable dans la vie quotidienne des humains. Autre particularité de taille: le régime d'échange des Wayuu est fondé sur ce que l'on peut appeler l'hétérosubstitution, à savoir l'interchangeabilité entre des personnes humaines et des richesses - surtout des têtes de bétail - tant pour les prestations matrimoniales que pour le prix du sang (Wergeld), une anomalie en Amérique du Sud ou l'homosubstitution (une personne humaine n'est échangeable que contre une autre personne humaine) règne sans partage, excepté chez les Mapuche du sud du continent qui adoptèrent également très tôt l'élevage extensif. Les Wayuu sont organisés en une trentaine de clans matrilinéaires fonctionnant comme des personnes morales, chacun associé à un animal (parfois plusieurs), sauvage en général, raison pour laquelle les ethnologues parlent à leur propos de clans «totémiques ». Or, l'examen du système segmentaire montre que cette qualification va au-delà de l'emploi classificatoire habituel du terme et qu'elle recouvre de fait une véritable ontologie totémique de type australien.

En premier lieu, il existe une identification profonde entre les segments humains et leurs troupeaux, laquelle est exprimée en termes de consubstantialité - la chair des membres d'un clan est réputée identique à celle de leurs animaux, ce qui apporte une amorce d'explication à la substituabilité des humains par les bovins dans les échanges. On note ensuite un parallélisme frappant dans les modes d'apparition des prototypes totémiques - les «grands-mères» (oushii) à l'origine des classes totémiques wayuu et les êtres du Rêve en Australie - les uns et les autres surgissant jadis de la terre dans des sites pour donner naissance aux lignées localisées dont les groupes totémiques sont l'expression. Le site d'émergence revêt une importance particulière pour les membres du patrilignage wayuu puisque c'est dans ses parages que l'on dépose les os des défunts, une opération qui est à rapprocher du fait qu'en Australie, l'âme d'un mort rejoint son site totémique afin de pouvoir se réincarner dans un autre individu. Plus important, enfin, est le fait que, dans les deux cas, les humains et les non-humains d'un groupe totémique partagent des qualités. En effet, de même qu'en Australie le nom d'un groupe totémique est souvent un nom de qualité servant à nommer l'animal éponyme et non un taxon zoologique, chez les Wayuu, le nom de clan ne renvoie pas à un animal ou à un lieu, mais à la caractéristique comportementale qui définit ses membres, laquelle est mentionnée pour chaque clan dans le mythe d'origine de cette institution ${ }^{5}$. En outre, et même si le nom de l'animal totémique n'est pas, comme en Australie, un nom de qualité, cet animal est néanmoins réputé exhiber de façon exemplaire la qualité particulière que les membres du clan sont dits avoir en commun.

5. Alessandro MANCUSO, «Descent among the Wayú. Concepts and social meanings», Journal de la société des américanistes, vol. 94, n 1, 2008, p. 99-126, note 11. 


\begin{tabular}{|l|l|}
\hline \multicolumn{1}{|c|}{ Australie } & \multicolumn{1}{c|}{ Wayuu } \\
\hline $\begin{array}{l}\text { Un prototype émerge de la terre au « temps } \\
\text { du Rêve », donnant origine à la classe } \\
\text { totémique. }\end{array}$ & $\begin{array}{l}\text { Une aïeule émerge de la terre il y a trois } \\
\text { générations donnant origine à la lignée } \\
\text { utérine. }\end{array}$ \\
\hline $\begin{array}{l}\text { Les groupes totémiques nommés regroupent } \\
\text { des humains et des non-humains ayant } \\
\text { les mêmes caractéristiques physiques } \\
\text { et morales. }\end{array}$ & $\begin{array}{l}\text { Les clans nommés sont dotés } \\
\text { d'une caractéristique comportementale } \\
\text { propre aux humains et aux animaux } \\
\text { éponymes. }\end{array}$ \\
\hline $\begin{array}{l}\text { Le nom du groupe est souvent dérivé } \\
\text { d'une qualité servant à nommer l'animal } \\
\text { éponyme. }\end{array}$ & $\begin{array}{l}\text { Le nom du clan est dérivé } \\
\text { de la caractéristique comportementale } \\
\text { de ses membres. }\end{array}$ \\
\hline $\begin{array}{l}\text { Identité ontologique des humains } \\
\text { et des non-humains du groupe totémique. }\end{array}$ & $\begin{array}{l}\text { Consubstantialité des humains } \\
\text { et des animaux domestiques permettant } \\
\text { à ces derniers de se substituer aux humains } \\
\text { dans les échanges. }\end{array}$ \\
\hline
\end{tabular}

Par contraste, le versant animiste du système wayuu est plus conforme aux régimes ontologiques dominants dans les basses terres d'Amérique du Sud. Pourtant, le rôle qu'y jouent les animaux domestiques et les rapports ambigus que les esprits maitres du gibier entretiennent avec eux l'est beaucoup moins. Un esprit féminin du nom de Pulowi est la maîtresse de tous les animaux, sauvages comme domestiques, terrestres comme marins, et elle se venge sur les humains des mauvais traitements qu'ils font subir aux uns comme aux autres, généralement en leur envoyant des maladies. Il n'y a ici ni échange ni négociation entre Pulowi ou ses émissaires et les chasseurs ou les pêcheurs, ces derniers traquant leurs proies à leurs risques et périls. Or, si la classification wayuu des animaux distingue bien entre sauvage et domestique, cette bipartition n'est en rien ontologique - ce ne sont pas des genres d'être différents et ils sont tous réputés autochtones; elle manifeste plutôt les différences dans leurs conditions respectives d'engendrement et dans la nature de ceux qui les contrôlent ${ }^{6}$. Le terme $u c h i i$ désigne la catégorie supragénérique des êtres vivant en collectifs indépendants des humains et sous le contrôle de Pulowi - les animaux sauvages, les prototypes d'espèce, les métapersonnes émissaires de Pulowi - tandis que mïrili englobe les animaux sous le contrôle des humains - les animaux domestiques, les animaux sauvages apprivoisés et les chiens. Selon la logique de l'animisme, les uns comme les autres vivent dans des collectifs spécifiques, sous l'autorité d'un puissant esprit maître pour les premiers, d'un esprit maître secondaire et d'un humain, pour les seconds.

Un dernier aspect du rapport des Wayuu aux animaux permet de préciser la nature des collectifs au sein desquels ceux-ci se déploient et les relations que ces collectifs entretiennent avec les collectifs humains. Il s'agit d'une catégorie de maux affectant la naissance et l'enfance - accouchements difficiles, maladies infantiles, malformations congénitales - transmises par contact avec des animaux, sauvages comme domestiques, et que Michel Perrin appelle «la contamination par les animaux ${ }^{7}$ ». La liste des

6. Michel PERRIN, «Creaciones míticas y representación del mundo: el ganado en el pensamiento simbólico guajiro », Antropológica, vol. 67, 1987, p. 3-31.

7. Michel PERRIN, « Les fondements d'une catégorie étiologique. (La notion de contamination chez les Guajiro) », L'Ethnographie, vol. 96-97, 1985, p. 103-122. 
animaux responsables varie selon les localités en sorte que toute la faune de la Guajira est potentiellement contaminante. L'effet pathogène est toujours dérivé d'une caractéristique physique ou éthologique de l'animal incriminé, la cure consistant à faire absorber par la personne affectée une partie d'un animal de l'espèce contaminante. Or, l'on peut identifier dans cette thérapeutique l'une des constantes du traitement animiste des animaux par les Amérindiens des basses terres de l'Amérique du Sud tropicale. Dans les collectifs animistes, en effet, les prohibitions et prescriptions alimentaires ont moins pour but de prévenir ou de favoriser la circulation de substances que d'empêcher ou de rendre possible le transfert depuis l'espèce interdite ou prescrite de traits anatomiques ou de modes de comportement dérivés de ces traits. Or, ce sont précisément ces caractéristiques physiques qui définissent les critères de recrutement des collectifs animistes, ces ensembles monospécifiques d'êtres qui s'assimilent à des tribus-espèces. La plupart des genres de non-humains ayant une intériorité analogue à celle des humains, ils se distinguent entre eux, et des humains, non pas leur «âme », mais par leur corps, c'est-à-dire par les dispositions qu'ils mobilisent pour actualiser le segment du monde que leur conformation physique les prédispose à habiter. À cela s'ajoute le fait que, partout dans l'archipel animiste, les personnes animales transmettent des afflictions aux personnes humaines si ces derniers ne prennent pas les précautions adéquates. L'une des manières communes de se prémunir contre ce danger est précisément la stratégie adoptée par les Wayuu, à savoir emprunter les dispositions physiques de l'espèce contaminante, par ingestion ou contact, afin de se faire reconnaître comme un congénère et neutraliser ainsi les effets de l'intromission accidentelle d'un corps humain dans l'altérité des corps d'une autre tribu-espèce. Chez les Wayuu, le régime animiste de la relation aux animaux ne se borne donc pas aux rapports ambigus et périlleux avec les esprits maîtres du gibier et à la totémisation du bétail; animaux sauvages comme animaux domestiques s'assemblent dans des collectifs avec lesquels les humains doivent composer, même pour ce qui est du cheptel dont la permanence dans la domestication n'est jamais assurée.

L'articulation entre animisme et totémisme chez les Wayuu se superpose ainsi à ces deux formes contrastées de relation entre humains et non-humains que sont la prédation et l'élevage. Que l'interaction avec les animaux sauvages soit pensée selon une logique animiste n'est pas pour surprendre ; c'est le cas pour tous les collectifs amérindiens des basses terres tropicales dans lesquels le gibier est doté de qualités et de comportements analogues à ceux ayant cours dans le monde humain. Dans la Guajira, comme dans d'autres régions de l'archipel animiste où la prédation domine, la chasse est un commerce périlleux avec un monde d'êtres et d'esprits qui se payent sur les humains des pertes que ceux-ci infligent dans leurs rangs. La maîtresse des animaux, Pulowi, est là pour protéger ses ouailles des prélèvements que les humains veulent effectuer, remplissant en cela la fonction partout dévolue au maître des animaux. Même si la chasse a pratiquement disparu dans les faits, l'existence de Pulowi ne fait aucun doute pour les Wayuu contemporains, pas plus que son désir toujours vivace de se venger sur les humains de ce qu'ils ont pu faire subir jadis aux animaux dont elle a la garde. Et c'est cette réalité attribuée à la maîtresse du gibier, à ses émissaires, à ses avatars et à ses intentions malveillantes qui permet de parler ici d'animisme.

En revanche, il peut paraître paradoxal de voir l'élevage comme organisé par un régime totémique, sauf si l'on considère que celui-ci ne définit pas à proprement parler le statut ontologique du bétail, mais son inclusion comme un enjeu dans des rapports sociaux caractérisés par un système segmentaire de clans totémiques. 
Certes, tout comme le gibier, le bétail possède des caractéristiques animistes. Celles-ci, toutefois, sont atténuées par rapport à celles du gibier. D'abord parce que les relations avec les animaux domestiques sont moins dangereuses que celles avec les animaux sauvages : contrairement aux chasseurs excessifs, l'éleveur qui maltraite son bétail ne risque pas d'être soustrait au monde des humains. Et si la menace de contamination que laissent planer les animaux domestiques existe bel et bien, son incidence est sans doute moins grave que celle dont les animaux sauvages sont responsables. En second lieu, le maître des animaux domestiques, Soleil, possède un statut bien différent de celui de la maîtresse du gibier. Son importance dans le monde des métapersonnes wayuu est très secondaire; il est au premier chef un être mythologique, sans incidence directe dans la vie des hommes. Contrairement à Pulowi dont la présence immanente se fait quotidiennement sentir pour tous les Wayuu, qu'ils soient chasseurs ou non, Soleil est une hypostase abstraite. En revanche, l'usage des animaux domestiques dans la vie sociale répond plus proprement à une logique totémique pour les raisons qui ont été exposées : en premier lieu l'identification entre les matrilignages et leurs troupeaux exprimés en termes de consubstantialité ; ensuite, la pratique de marquer le bétail au fer avec des emblèmes figurant l'animal totémique du clan, manière d'incorporer les animaux domestiques, non pas tant comme des éléments non humains du clan totémique, à l'instar de ce qui se passe en Australie, que comme des indices vivants de son existence concrète et de sa permanence dans le temps. L'incorporation des animaux domestiques dans la logique totémique des clans est enfin très visible dans le rôle qu'ils jouent comme substituts des humains, aussi bien dans l'alliance matrimoniale que dans la compensation des homicides. Mais ce rôle est plus manifeste encore dans une autre fonction de substitution, la dispersion du troupeau des défunts. Tous les animaux domestiques d'un mort sont distribués par ses proches parents masculins au cours des deux phases de son enterrement et il est très fréquent que le troupeau ne suffise pas, certains membres de la famille devant puiser dans leur cheptel personnel pour que chaque personne présente à l'enterrement reparte avec un animal. L'équivalence entre le clan et son bétail est ici transparente : quand le groupe perd l'un de ses membres, il faut que disparaissent avec lui ce qui a été son moyen de vie, son statut social, ce par quoi il existait et contribuait à l'existence de ce groupe. On peut donc avancer une hypothèse : si les animaux totémiques traditionnels ont perdu leur importance comme signe d'identification des clans (les listes proposées par les observateurs depuis plus d'un siècle sont contradictoires entre elles), c'est parce que le bétail remplit à présent cet office. L'incertitude quant aux animaux totémiques correspondant à chaque clan pourrait simplement signifier que la fonction d'identité autrefois attribuée à l'animal éponyme a été transférée aux animaux domestiques : marqué du signe d'un animal totémique, le bétail porte en lui et sur lui l'identité du clan et du matrilignage; bien qu'il ne figure pas dans les récits étiologiques des clans totémiques, il a été en quelque sorte totémisé afin de symboliser la segmentarité wayuu dont il assure le fonctionnement effectif.

\section{Les déclinaisons de l'animisme en Asie du Sud-Est}

Après avoir examiné les combinaisons structurelles entre animisme et totémisme, on s'est tourné vers des formes apparentes d'hybridation entre animisme et analogisme choisies en Asie du Sud-Est où elles sont fort communes. Si l'animisme s'appuie sur un dualisme tranché de l'intériorité et de la physicalité, l'analogisme 
prend acte du fait que le monde est composé d'une infinité d'éléments, d'états, de situations, de qualités, d'êtres, tous différents les uns des autres, qu'il faut pouvoir connecter dans des correspondances au moyen de l'analogie, raison pour laquelle on a choisi d'appeler «analogiste» ce régime ontologique. S'opposent ainsi deux façons contrastées de détecter et de systématiser des ressemblances et des différences, lesquelles aboutissent, dans le cas de l'animisme, à une continuité morale entre humains et non-humains et à une discontinuité de leurs dimensions physiques, dans le cas de l'analogisme à une double discontinuité, morale et physique, que des réseaux de correspondances s'efforcent en vain de rendre continue. Or, en Asie du Sud-Est tant continentale qu'insulaire, l'animisme sous sa forme paradigmatique est rare et l'on trouve plutôt des formes mixtes où sont présents des éléments tant de l'animisme que de l'analogisme. À la différence des cas d'articulation entre animisme et totémisme examinés auparavant et qui reviennent tous à une superposition de deux grilles ontologiques distinctes s'appliquant alternativement aux mêmes objets selon leur type de mobilisation dans la vie sociale, la combinaison entre animisme et analogisme dans cette partie de l'Asie prend plutôt l'apparence d'un continuum de collectifs qui exhibent de plus en plus de traits analogistes et de moins en moins de traits animistes, ou vice versa selon le point de départ que l'on adopte. Il importait donc d'établir une typologie fine des formes de transitions de l'animisme à l'analogisme en examinant ce qui change à chaque palier de la transformation dans la nature des éléments composant les collectifs.

Le premier bloc, caractéristique de ce que l'on pourrait appeler l'animisme standard, très minoritaire en Asie du Sud-Est, est constitué par des populations tels les groupes aborigènes connus sous le nom d'Orong Asli en Malaisie (Chewong, Ma'Betisék, Batek...) ou les Penan de Bornéo, qui présentent tous les traits de l'animisme amazonien ou sibérien : la plupart des non-humains ont une intériorité clairement singularisée analogue à celles des humains, mais chaque classe d'existant se distingue par son corps conçu comme un vêtement. Le perspectivisme est très commun, tant chez les humains que chez les animaux et les esprits, lesquels ne se voient pas eux-mêmes tels que les autres les voient tout en voyant les autres de façon différente que ceux-ci ne se voient eux-mêmes. La différence des physicalités - chaque genre d'être est revêtu d'un corps sui generis - fonde la distribution des habitants du cosmos entre des tribus-espèces d'humains et de non-humains situées sur un pied d'égalité. Enfin, les rapports entre humains et non-humains sont fondés sur la prédation réciproque mise en œuvre pour les premiers principalement dans la chasse et, pour les seconds, au moyen de la vengeance que les esprits protecteurs du gibier exercent sur les humains en leur envoyant des maladies.

Un deuxième bloc est constitué par la majorité des populations tribales d'Asie du Sud-Est qui vivent en général dans les collines, à l'écart des plaines où sont concentrés les centres urbains, dans cette région que l'on a pris coutume d'appeler Zomia ${ }^{8}$ et qui désigne les hautes terres de l'Asie du Sud-Est continentale occupées par des groupes ethniques qui sont parvenus jusqu'à très récemment à échapper en partie au contrôle des États coloniaux puis postcoloniaux implantés dans les basses terres. Bien que Zomia n'englobe pas l'Asie du Sud-Est insulaire et péninsulaire, on retrouve aussi en Indonésie, en Malaisie et aux Philippines le type de contraste que cette notion met en

8. James ScotT, The Art of Not Being Governed. An Anarchist History of Upland Southeast Asia, New Haven/Londres, Yale University Press, coll. « Agrarian Studies », 2009. 
évidence, en particulier le contraste entre les centres du pouvoir économique et politique situés sur le littoral et à l'embouchure des fleuves, et les groupes tribaux de l'amont et des hautes terres. On a choisi d'appeler cet ensemble «les montagnards », comme l'on fait les Français en Indochine pour désigner les populations de la cordillère annamitique, une dénomination qui correspond bien à l'expression «hill-tribes » employée en anglais pour les désigner. Quelles sont les caractéristiques de la cosmologie montagnarde ? Elle est portée par des populations qui pratiquent la culture sur essart du riz pluvial et l'élevage du cochon et du buffle, et pour qui les animaux domestiques ont remplacé les animaux sauvages en termes de partenaires sociaux, économiques et rituels. Le premier trait marquant qui rapproche les cosmologies montagnardes des régimes ontologiques animistes est la prolifération des esprits : esprits du lieu, maîtres des animaux et des plantes, ancêtres ou fantômes sont généralement coiffés par un esprit dominant et organisés dans une gradation plus ample comprenant les autres êtres, animés et inanimés, dont les humains, ces derniers le plus souvent insérés dans une position intermédiaire entre les esprits du monde céleste et ceux du monde chthonien. Par contraste avec les formes d'animisme typiques de l'Amazonie ou de l'Amérique du Nord septentrional, cette prolifération d'esprits est ainsi hiérarchisée et les relations entre les strates sont entretenues par l'intermédiaire du sacrifice. Les humains offrent des animaux domestiques, idéalement des buffles, aux ancêtres et aux esprits les plus puissants qui sont les maîtres de la terre, de la forêt et des eaux, en échange du droit d'utiliser les ressources dont ils sont les gardiens.

Pour illustrer ce deuxième bloc on s'est attardé sur l'exemple des Katou, bien décrits dans les publications d'Yves Goudineau et de Kaj Århem ${ }^{9}$. Cette population des hautes terres du Vietnam central et du Laos mobilise l'assistance des bons esprits, pour l'essentiel des ancêtres, afin qu'ils servent de médiateur auprès des puissants esprits de la forêt qui ont tous la capacité d'infliger des dommages. Le sacrifice est le principal moyen pour se concilier leur bonne grâce car les animaux domestiques élevés par les humains sont leur nourriture principale. Satisfaire les esprits en les nourrissant permet de maintenir l'ordre cosmique, d'éviter les afflictions, de racheter les fautes et de restaurer l'ordre social. Car s'ils sont négligés ou offensés, les esprits se mettent en colère et dévorent les âmes des humains, causant ainsi la maladie et la mort. Les Katou sont par ailleurs des chasseurs enthousiastes et ils disent du gibier qu'il est le cheptel des esprits de la forêt ayant pour mission de veiller sur lui, une conception très commune dans tout l'archipel animiste. Le plus important de ces maîtres des animaux sauvages, une figure féminine appelée Komorbarr, est réputé livrer des individus de son troupeau aux humains par compassion pour eux, un don témoignant d'une attitude de générosité bienveillante qui doit être reconnue et célébrée par les villageois katou au moyen d'un rituel lorsqu'une chasse est couronnée de succès. Au cours des différentes opérations cérémonielles, la viande du gibier est traitée comme la viande des animaux domestiques sacrifiés : elle est offerte aux esprits avant d'être consommée en commun par les villageois. Le parallélisme entre sacrifice et rituel de chasse est toutefois plus

9. Kaj ÅRHEM, «Southeast Asian animism in context» et «Animism and the hunter's dilemma. Hunting, sacrifice and asymetric exchange among the Katu of Vietnam » in K. Århem et G. Sprenger (dir.), Animism in Southeast Asia, Londres, Routledge, 2016, p. 3-30 et p. 91-113 ; Yves GoudineaU, "L'anthropologie du Sud-Laos et l'origine de la question Kantou », in Y. GOUDINEAU et M. LORRILLARD (dir.), Recherches nouvelles sur le Laos, Vientiane/Paris, École française d'Extrême-Orient, 2008, p. 639-664. 
complexe en ce que le second dédouble les opérations et les protagonistes du premier. En effet, le rituel de chasse porte sur un animal sauvage dont le sacrifiant n'est pas un humain, mais un esprit, et dont le destinataire, du moins dans un premier temps, n'est pas une communauté d'esprit, mais une communauté d'humains. Car c'est la maîtresse du gibier, Komorbarr, qui est la vraie sacrifiante initiale, la donatrice de l'animal aux humains, le chasseur n'étant que le sacrificateur, l'instrument occasionnel de la mort. En revanche, le repas rituel des villageois constitue le retour aux esprits de l'offrande qui a d'abord été faite aux humains, selon la logique sacrificielle classique qui veut que ces derniers mangent la chair dont ils offrent l'odeur aux esprits. Le parallélisme entre animal sauvage et animal domestique fonctionne aussi dans l'autre sens puisque le buffle, avant d'être sacrifié sur la place du village, est attaqué à coups de lance comme s'il était chassé. L'exemple des Katou, qui s'arrangent pour transformer en une opération sacrificielle un rite animiste d'échange entre les humains et les esprits, montre combien le sacrifice est central dans cette forme d'animisme propre à l'Asie du Sud-Est. Il exprime la dépendance des humains envers les esprits, mais aussi la confiance placée en leur bienveillance et l'attente de leurs bienfaits. En contraste avec l'intersubjectivité symétrique des relations entre humains et animaux caractérisant l'animisme standard dans les Amériques ou en Sibérie, l'intersubjectivité qui se déploie dans l'animisme sacrificiel de l'Asie du Sud-Est est fondamentalement asymétrique et se trouve centrée, non plus sur les relations des humains avec des animaux ayant les propriétés des esprits, mais avec des esprits que l'on nourrit des animaux élevés par les humains à leur profit.

Un dernier trait des ontologies montagnardes a été bien mis en lumière par Jeanne Cuisinier dans son classique Sumangat. L'âme et son culte en Indochine et en Indonésie ${ }^{10}$. On trouve dans toute la région l'idée que le monde est animé par un principe, souvent glosé comme une âme, qui insuffle en ses composantes une capacité d'agir, donc une sorte d'intériorité universelle et distribuée. Or, ce principe de vie est réparti de façon inégale ; il s'agit d'une disposition qui qualifie chaque être, animé ou inanimé, mais qui se manifeste avec une intensité plus ou moins grande. En outre, cette disposition subjective peut non seulement prendre la forme d'une âme individuelle, comme c'est le cas dans l'animisme standard, elle peut aussi être vue comme une capacité à agir qui trouve sa source à l'extérieur de l'être qui lui est associé et qui prend souvent la forme d'une qualité personnelle hypostasiée dans un esprit: le courage, l'ambition, la ruse, la force de caractère. Tant comme un aspect de l'intériorité que comme un attribut provenant de l'extérieur et conféré par un esprit, cette puissance tend à être réifiée comme substantielle - une essence concrète, un foyer d'énergie - à la fois détachable de l'être qu'elle anime et pouvant se matérialiser dans des objets qui deviennent à la fois des signes de richesse et des signes de puissance morale : les gongs, les tambours de bronze, les grandes jarres de terre cuite, les tissus brodés. C'est parce que l'âme universelle est en mesure de recevoir une forme substantive et visible dans un être qu'elle peut être différenciée et quantifiée, en sorte que la conception de la personne dans les tribus montagnardes a pu être définie avec justesse comme une «théorie des âmes inégales » ${ }^{11}$.

10. Jeanne CUISINIER, Sumangat. L'âme et son culte en Indochine et en Indonésie, préface de Louis Massignon, Paris, Gallimard, 1951.

11. A. Thomas KIRSCH, Feasting and Social Oscillation: Religion and Society in Upland Southeast Asia, Ithaca (New York), Cornell University Press, 1973, p. 24. 
L'inégalité des âmes s'accentue fortement dans le dernier degré du continuum animiste d'Asie du Sud-Est, celui des collectifs très hiérarchisés que l'on a illustré avec le cas des Toradja de l'île de Célèbes. Leur ontologie a ceci de particulier qu'elle est composée de classes d'êtres fortement différenciés, y compris parmi les humains, puisqu'ils furent forgés par le démiurge à partir de matériaux distincts (or, fer, boue, pierre, canne à sucre). Trois humanités coexistent ainsi au sein de l'ensemble Toradja - les nobles, les gens du commun et les esclaves - dont la «nature » (rupa) est incommensurable. Voisins des Toradja au sud de Célèbes, et pourtant islamisés depuis le XVII ${ }^{\mathrm{e}}$ siècle, les Bugis ont le même genre de cosmologie hiérarchique fondée sur une gradation de la puissance cosmique qui résulte, là aussi, d'un caractère physique : les nobles sont nés avec une quantité importante de « sang blanc » qui leur a été transmis par filiation depuis des esprits ancestraux. Par leur naissance, ils forment donc une espèce à part, la potentialité physique qu'ils recèlent devant par ailleurs être entretenue et cultivée. La distribution différentielle de pouvoir cosmique entre les existants est ainsi la base de la hiérarchie qui les ordonne. Bref, chez les Toradja comme chez les Bugis, l'inégalité des intériorités est fondée paradoxalement sur la répartition différentielle des physicalités.

Quelles conséquences cela entraîne-t-il sur la redéfinition de l'animisme, en premier lieu sur cette caractéristique élémentaire qu'est l'attribution d'une subjectivité aux non-humains, en particulier aux animaux ? Il semble bien que, contrairement à ce qui se passe dans d'autres parties de l'archipel animiste, en Amazonie, en Amérique du Nord, en Sibérie ou même, plus à l'ouest, chez les Chewong ou les Ma'Betisék, les plantes et les animaux du monde toradja - à l'exception du buffle, du riz et du chat - ne sont pas dotés d'une subjectivité individuelle, cette qualité étant réservée à leurs ancêtres au moment où ils avaient encore forme humaine. Cela dit, les animaux et les plantes sont dits avoir des «esprits de vie»(deata), lesquels sont moins conçus comme des subjectivités individuelles que comme des manifestations de l'intériorité toujours agissante de l'ancêtre de l'espèce. On peut donc considérer qu'il existe chez les Toradja deux modalités d'animation plus ou moins particularisées : les esprits de vie deata liés à des corps particuliers et la puissance cosmique sumanga, au fondement de toute animation et s'exprimant dans des phénomènes qui n'ont pas toujours une forme discrète. Il s'agit ainsi d'un animisme plus diffus que celui qui a cours dans d'autres régions de l'archipel animiste puisqu'il se déploie le long d'un continuum dans lequel, si certains non-humains ont bien le même type d'intériorité que les humains, la majorité des autres existants - des organismes jusqu'aux cours d'eau en passant par les maisons ou les talismans - sont plutôt des expressions plus ou moins particularisées d'une énergie cosmique distribuée en eux à des degrés divers de concentration. L'autre dimension de l'animisme, à savoir que chaque classe d'existant possède une physicalité distincte, est aussi présente, puisque les types d'êtres de la cosmologie toradja se différencient tous par des « natures » distinctes (rupa), y compris au sein des humains. En revanche, les collectifs en forme de tribuespèce typiques de l'animisme ne semblent pas avoir cours chez les Toradja, remplacés qu'ils sont par une sociabilité cosmique reliant des êtres de toutes sortes dans un même réseau d'interdépendances, un trait classique des collectifs analogistes.

Par ailleurs, le «multinaturalisme » toradja prend un aspect singulier, notamment pour ce qui est des humains, en ce qu'il est très hiérarchisé. Faits de substances différentes, les trois types d'humains sont répartis en trois castes que les Toradja appellent des «pieux »: les pieux d'or (les nobles), les pieux de fer (les gens du 
commun) et les pieux du nœud de la canne à sucre (les esclaves). Les matières placées au cœur du mélange réalisé par le démiurge lorsqu'il a créé les trois sortes d'humains sont réputées se transmettre dans le sang maternel de génération en génération, conservant ainsi la nature différente de chaque caste au fil du temps. Parler de caste n'est pas indu dans le cas présent, car non seulement leurs membres ont des corps différents, mais leurs fonctions, bien que hiérarchisées, sont complémentaires, notamment dans l'organisation du sacrifice. Or, les différences de corps entre les trois castes correspondent tout à fait aux genres de différence dans les dispositions physiques qui caractérisent les discontinuités entre tribus-espèces dans l'animisme. Ces différences physiques sont ici indexées sur la pureté du sang : plus l'on monte dans la hiérarchie, plus le sang est pur, et plus l'on est beau, épris de vérité et impénétrable. C'est la raison pour laquelle une ségrégation stricte est maintenue entre les castes : le sang pur des nobles de plus haut rang ne tolère pas le sang impur des esclaves. Par rapport à l'animisme «standard », le modèle toradja présente donc une double inflexion. L'intériorité des existants humains et non humains n'y prend pas la forme habituelle d'une âme ou d'un esprit nettement individualisé ; elle est plutôt la diffraction en chacun d'eux d'une puissance vitale qui anime le cosmos tout entier. Toutefois, ces manifestations de l'âme cosmique ne sont pas homogènes ou isonomiques ; non qu'elles refléteraient chacune une position différente dans le monde, ou une trajectoire biographique particulière, comme c'est le cas dans l'animisme standard. La singularisation des intériorités vient de ce que chaque classe d'être incorpore et exprime la subjectivité universelle à des degrés très divers selon la concentration d'essence cosmique qu'elle a reçue en partage.

L'autre inflexion du système toradja par rapport à l'animisme ordinaire concerne la physicalité. Comme c'est le cas ailleurs dans l'archipel animiste, chaque classe d'être possède bien des dispositions physiques qui lui sont propres et qui différencient cette classe des autres par les actions que ces dispositions permettent à ses membres d'accomplir dans un milieu donné, une sorte d'éthogramme fournissant la base à partir de laquelle ses membres peuvent constituer une tribu-espèce sui generis. Or, on se souviendra que cette fragmentation des natures, chacune propre à une classe d'existant, affecte aussi les humains. Dans l'animisme ordinaire, l'humanité ne constitue pas un ensemble homogène, mais se voit dissociée en un grand nombre de tribus-espèces, chacune se différenciant de ses voisines par son habitat, ses habitudes alimentaires, ses parures et son costume, ses armes et ses outils, la langue qu'elle parle et l'ethos qu'elle manifeste dans son comportement. Chaque collectif humain se distingue donc des autres alentour par un corps propre et une manière de s'en servir. Or, ce qui change dans le cas toradja, c'est que certaines tribus-espèces d'humains, au lieu de mener une existence autonome, se retrouvent intégrées à l'intérieur d'un collectif dont elles constituent une strate hiérarchisée. Chaque caste forme ainsi une tribu-espèce indépendante des autres castes sur le plan ontologique, mais dépendante d'elles sur les plans fonctionnel et liturgique. Le multinaturalisme typique de l'animisme est donc bien maintenu, mais redéployé et hiérarchisé à l'intérieur même des collectifs au lieu d'être, comme dans l'animisme standard, la marque visible de la différence entre les collectifs. Il en résulte une double différenciation: des subjectivités à l'intérieur du cosmos en fonction du degré de densité de la puissance cosmique que chaque classe d'être est en mesure de réfléchir, et des physicalités de chaque classe d'être puisque les corps sont hiérarchisés en nature selon ce qu'il leur est loisible d'accomplir. Cette double différenciation constitue ainsi comme une amorce de la fragmentation générale des intériorités et des physicalités typique du 
régime ontologique de l'analogisme, le cas toradja occupant une position cruciale au sein d'un continuum de transformations propre à l'Asie du Sud-Est, à la jonction des formes les plus distribuées d'animisme et des formes les plus intersubjectives d'analogisme. C'est ce que le tableau suivant permet d'apprécier.

\begin{tabular}{|c|c|c|c|}
\hline & $\begin{array}{l}\text { Animisme } \\
\text { standard } \\
\text { (Chewong, Penan, } \\
\text { etc.) }\end{array}$ & $\begin{array}{l}\text { Animisme distribué } \\
(\ll \text { montagnards })\end{array}$ & $\begin{array}{l}\text { Animisme stratifié } \\
\text { (collectifs à castes) }\end{array}$ \\
\hline $\begin{array}{l}\text { Type } \\
\text { d'intériorité }\end{array}$ & $\begin{array}{l}\text { Humains et non- } \\
\text { humains ont une } \\
\text { intériorité } \\
\text { nettement } \\
\text { individualisée. }\end{array}$ & $\begin{array}{l}\text { Intériorité diffractée } \\
\text { («âme du monde »), } \\
\text { réifiable et présente en } \\
\text { chaque être à des degrés } \\
\text { inégaux d'intensité. }\end{array}$ & $\begin{array}{l}\text { Intériorité diffractée, } \\
\text { réifiable et présente } \\
\text { en chaque être à des } \\
\text { degrés inégaux en } \\
\text { fonction des } \\
\text { différences de } \\
\text { physicalité ; absente } \\
\text { dans la plupart des } \\
\text { plantes et des } \\
\text { animaux. }\end{array}$ \\
\hline $\begin{array}{l}\text { Type de } \\
\text { physicalité }\end{array}$ & $\begin{array}{l}\text { Chaque « espèce » } \\
\text { d'humains et de } \\
\text { non-humains a un } \\
\text { corps qui lui est } \\
\text { propre (vêtement). }\end{array}$ & $\begin{array}{l}\text { Les capacités d'agir des } \\
\text { humains et des non- } \\
\text { humains varient selon le } \\
\text { degré de concentration de } \\
\text { leur intériorité. }\end{array}$ & $\begin{array}{l}\text { Les capacités d'agir } \\
\text { sont toutes } \\
\text { différentes, y compris } \\
\text { au sein des collectifs } \\
\text { humains, du fait de } \\
\text { leurs «natures » } \\
\text { distinctes. }\end{array}$ \\
\hline $\begin{array}{l}\text { Type de } \\
\text { perspective }\end{array}$ & $\begin{array}{l}\text { Généralisé pour } \\
\text { tous les humains } \\
\text { et non-humains. }\end{array}$ & $\begin{array}{l}\text { Plutôt restreint aux } \\
\text { humains et aux esprits. }\end{array}$ & $\begin{array}{l}\text { Exclusivement } \\
\text { restreint aux humains } \\
\text { et aux esprits. }\end{array}$ \\
\hline $\begin{array}{l}\text { Type de } \\
\text { collectifs }\end{array}$ & $\begin{array}{l}\text { Humains et non- } \\
\text { humains vivent } \\
\text { dans des tribus- } \\
\text { espèces égalitaires. }\end{array}$ & $\begin{array}{l}\text { Les collectifs humains } \\
\text { sont égalitaires, les } \\
\text { collectifs d'esprits ne le } \\
\text { sont pas. }\end{array}$ & $\begin{array}{l}\text { Les collectifs } \\
\text { humains et non } \\
\text { humains sont tous } \\
\text { hiérarchisés. }\end{array}$ \\
\hline $\begin{array}{l}\text { Type de } \\
\text { relation entre } \\
\text { humains et } \\
\text { non-humains }\end{array}$ & $\begin{array}{l}\text { Chasse et } \\
\text { horticulture sur } \\
\text { brûlis } \\
\text { embryonnaire } \\
\text { Prédation } \\
\text { réciproque. }\end{array}$ & $\begin{array}{l}\text { Chasse, polyculture, } \\
\text { riziculture pluviale, } \\
\text { élevage. } \\
\text { Échanges et dons passant } \\
\text { par le sacrifice. }\end{array}$ & $\begin{array}{l}\text { Chasse résiduelle, } \\
\text { riziculture pluviale et } \\
\text { inondée, élevage. } \\
\text { Échange et dons } \\
\text { passant par le } \\
\text { sacrifice médiatisé } \\
\text { par un spécialiste } \\
\text { rituel. }\end{array}$ \\
\hline
\end{tabular}

Quelles sont les conditions du passage d'un type d'animisme à l'autre ? L'élément principal qui transforme l'animisme standard en animisme distribué est le passage d'une intériorité de même type chez les humains et de nombreux non-humains à une intériorité graduée, c'est-à-dire réfléchissant de façon inégale en chaque être la subjectivité généralisée que les spécialistes de la région appellent puissance cosmique ou âme du monde. Il ne s'agit pourtant pas encore d'une hiérarchie à proprement parler, mais d'une diversité des façons d'appréhender la personne humaine et non humaine, celle-ci variant par ses aspects plutôt que par la nature de ses composantes, en sorte qu'il y a autant d'aspects que d'objets avec lesquels un sujet quelconque établit 
une relation singulière à un terme singulier ${ }^{12}$. Ainsi, et dans la mesure où la gradation des intériorités est un effet de l'intensité des relations dans lesquelles les personnes humaines et non humaines s'engagent en fonction de leur interlocuteur, l'imputation d'une subjectivité à un être est moins la conséquence de la reconnaissance en lui d'un attribut intrinsèque - une «âme » ou une faculté langagière - que l'effet du type d'interaction avec cet être que telle ou telle circonstance autorise.

Le passage de l'animisme distribué à l'animisme stratifié résulte, quant à lui, d'un double mouvement. Il se produit d'abord une stabilisation des intériorités graduées dans une gamme de physicalités qui leur correspondent, des «natures », lesquelles étaient de toute façon déjà différentes, comme elles le sont toujours en régime animiste. Et cette stabilisation d'âmes inégales dans des physicalités elles-mêmes toutes distinctes ne découle plus des accidents de la vie ou de la variabilité des talents personnels chez les humains, comme c'était le cas dans l'animisme distribué, mais d'une cosmogénèse au cours de laquelle sont créées $a b$ initio des classes d'êtres ontologiquement différents, y compris parmi les humains. Par contraste avec les mythes d'origine de l'animisme standard, amazonien ou sibérien, l'ontogenèse de l'animisme stratifié n'est plus un processus de spéciation, c'est-à-dire un passage catastrophique du continu au discret, mais un processus d'emblée créatif de fabrication progressive de classes d'êtres qui sont dès le début tout à fait distinctes tant par leurs physicalités que par leurs intériorités. Une fois accompli ce double mouvement de différenciation, les humains disséminés en tribus-espèces se trouvent disponibles pour être regroupés à l'intérieur d'un même collectif en une pyramide de castes, à l'instar des collectifs toradja ou bugis. Que ce mouvement se soit empiriquement produit par divisions internes d'un collectif humain égalitaire ou par agrégation à l'intérieur d'un même collectif de groupes au départ étrangers les uns aux autres, seule l'histoire, lorsqu'elle est accessible, peut le dire au cas par cas. Mais peu importe en définitive, car c'est la logique structurelle du mécanisme qui importe ici et celle-ci pointe clairement vers l'émergence de la hiérarchie, ce dispositif d'ordonnancement des différences si central en régime analogiste. Et pourtant, ces collectifs à castes sont encore animistes car les genres d'existant dont ils sont faits ont une subjectivité qui, pour être échelonnée en degrés d'intensité, n'est pas pour autant tout à fait fragmentée pour chacun d'entre eux, comme elle l'est dans les ontologies analogistes.

\section{Passage de l'animisme à l'analogisme en Asie du Sud-Est}

C'est ainsi de manière presque insensible que s'opère le passage de l'animisme stratifié à l'analogisme proprement dit, c'est-à-dire le basculement d'une ontologie dans une autre. Entre ces deux modes d'identification, en Asie du Sud-Est du moins, la différence est de degrés, non de nature. Elle s'exprime de deux façons. D'abord par une extension considérable de la hiérarchie sociocosmique et une augmentation du nombre de ses composantes, humaines et non humaines; ensuite, par un recours systématique aux réseaux de correspondances analogiques afin de relier entre elles toutes ces singularités nouvelles. Étagement hiérarchique et recherche de correspondances, les méthodes les plus communes employées dans les ontologies

12. Guido SPRENGER, « Graded personhood: Human and non-human actors in the Southeast Asian uplands », in K. ÅrHem et G. SPREnGER (dir.), Animism in Southeast Asia, Londres, Routledge, 2016, p. 38. 
analogistes afin de faire tenir ensemble les éléments disparates formant le monde deviennent ainsi beaucoup plus visibles que dans les variantes mêmes les plus stratifiées d'animisme. C'est ce que l'exemple des Shan a permis d'établir.

Dispersés dans une multitude de poches depuis l'Assam et le sud de la Chine jusqu'à la Birmanie et au Tonkin, les Shan se sont longtemps organisés dans de petites principautés assez autonomes, occupant les fonds de vallées où ils pratiquent la riziculture irriguée, en interaction continue avec les tribus montagnardes qui vivent dans les collines de la culture sur essart du riz pluvial. En dépit de ce peuplement en mosaïque intercalé entre des populations de langues et de cultures différentes, généralement animistes, les Shan présentent une grande homogénéité et l'on peut à bon droit les considérer comme une illustration de la forme la plus commune d'analogisme en Asie du Sud-Est. Cela tient en premier lieu à ce que leurs collectifs sont plus hiérarchisés que ceux des systèmes à castes. Certes, la segmentation shan ressemble à celle des Toradja ou des Bugis, avec une répartition tripartite entre une noblesse héréditaire, un bloc intermédiaire d'agriculteurs et un conglomérat de gens formant les classes les moins considérées, à quoi s'ajoutaient jadis des esclaves. À l'intérieur de ces trois ordres, toutefois, les subdivisions de fait sont multiples. Tout en haut de la pyramide, le saohpa est un roi sacré régnant sur une aristocratie qui entretient avec lui des liens de parenté et dont les membres sont classés par ordre d'importance selon leur proximité au souverain et le degré de dignité des fonctions qu'ils exercent. Le bloc des agriculteurs n'est différencié que par la plus ou moins grande aisance matérielle de ses membres, la richesse constituant chez les Shan la manifestation visible de la plénitude morale qu'un individu peut atteindre. Enfin, les basses classes sont très diversifiées: pêcheurs, bouchers, négociants d'alcool, gardiens de porcs, tous ceux qui accomplissent des tâches subalternes et impures selon le code bouddhiste, mais là encore subtilement stratifiés. À cette hiérarchie étendue d'humains correspond une hiérarchie étendue des non-humains. Les Shan ont beau être des bouddhistes theravada, ils cohabitent aussi avec une foule d'esprits également très sédimentés. Ces esprits sont souvent associés à des lieux - cours d'eau, vallées, forêts, maisons ; certains sont les gardiens des villages, d'autres sont associés aux quatre éléments, d'autres encore sont attachés à des tatouages et à des amulettes protectrices et veillent sur ceux qui les portent. Abondent en outre les fantômes, les monstres et les sorciers, également personnalisés et localisés. Naga, le serpent cosmique, et phii long, le grand esprit, sont aussi des métapersonnes avec lesquelles il faut compter. Enfin, et malgré la réticence du bouddhisme à l'égard du sacrifice, les Shan offrent des animaux aux esprits, notamment ceux qui protègent les villages, au cours de rites dont les motivations et le déroulement ne se démarquent guère de ceux de l'animisme distribué et de l'animisme stratifié. Bref, le monde shan fourmille de non-humains tout aussi échelonnés et singularisés par leurs fonctions et leurs sphères d'action que le sont les humains. En définitive, par rapport à l'animisme stratifié des Toradja, le cosmos analogiste shan ne se distingue que par un étagement plus feuilleté de la hiérarchie et par un mobilier ontique beaucoup plus abondant ; sur le plan structurel, le second pourrait être vu comme une diversification du premier.

En revanche, le système calendaire ${ }^{13}$ dont les Shan se servent notamment afin de déterminer les jours fastes et néfastes pour l'accomplissement de telle ou telle activité

13. Nicola TANnEnbaum, "Shan calendrics and the nature of Shan religion », Anthropos, vol. 19, 1984, p. 505-515. 
constitue un modèle de ces réseaux de correspondances que les systèmes analogistes emploient pour connecter des singularités, réseaux qui sont plus rares dans les régimes ontologiques animistes, même en Asie du Sud-Est. Le système shan est fondé sur le calendrier bouddhiste de douze mois lunaires, mais les unités constitutives et les valeurs dont elles sont affectées se voient déterminées par de nombreux autres facteurs : par exemple, le genre de nourriture que les esprits consomment selon les jours de l'année, une information fondamentale pour déterminer le type d'offrandes qui leur convient et les activités recommandées ou proscrites. Comme dans tout réseau de correspondances efficace, aucune singularité n'est ici laissée de côté : chaque moment, chaque point et orientation de l'espace, chaque événement, chaque type de relation, chaque tâche à accomplir, chaque tempérament sont connectés dans des associations signifiantes et pratiques afin de réduire l'incertitude qu'engendre pour les humains l'obligation de vivre dans le genre de monde infiniment diversifié dont les ontologies analogistes se plaisent à figurer les reflets.

\section{De l'animisme à l'analogisme : le rôle du sacrifice en Asie du Sud-Est}

Le continuum qui conduit de l'animisme standard des Chewong jusqu'à l'analogisme tout aussi standard des Shan n'est intelligible que si l'on prête attention à une suite de minuscules modifications portant sur la nature de l'intériorité détectée chez les humains et les non-humains, sur les rapports entre les spécificités de cette intériorité et les spécificités de la physicalité, sur le degré de diversification des composantes du cosmos et sur l'ampleur des mécanismes mis en œuvre pour le réduire. Il reste pourtant un point à éclaircir dans cette transition graduelle, c'est le rôle qu'y joue le sacrifice. À grands traits, on peut dire qu'il est très rare dans les collectifs relevant de l'animisme standard : absent parmi les populations qui refusent l'élevage, comme les Chewong, les Ma'Bétisek, les Batek ou les Penan, il est réduit à des offrandes rituelles de poulet ou de cochon parmi des populations comme les Bontoc ou les Buid. En revanche, le sacrifice est généralisé tant dans l'animisme distribué et l'animisme stratifié que dans l'analogisme standard où il se voit seulement limité par des versions rigoristes du bouddhisme et de l'islam qui ne sont pourtant pas parvenues à l'éliminer.

Ce constat contredit ainsi l'hypothèse avancée dans Par-delà nature et culture ${ }^{14}$ que le sacrifice est un dispositif de couplage typique de l'analogisme dans la mesure où il a pour finalité principale de relier deux termes entre lesquels il n'existe au départ aucun lien, le but de l'opération étant, pour reprendre la définition proposée par Lévi-Strauss, « d'instaurer un rapport, qui n'est pas de ressemblance, mais de contiguïté, au moyen d'une série d'identifications successives qui peuvent se faire dans les deux sens [...] : soit du sacrifiant au sacrificateur, du sacrificateur à la victime, de la victime sacralisée à la divinité ; soit dans l'ordre inverse ${ }^{15} \gg$. Employer le sacrifice pour forger une relation entre des entités initialement dissociées peut donc se révéler pertinent dans un régime ontologique où tous les existants sont des singularités ; cela permet d'établir un lien entre deux entités distantes et hétérogènes, le sacrifiant et le destinataire du sacrifice, au moyen d'identifications graduelles et transitives entre des éléments intermédiaires. Or, si le sacrifice est inconnu dans les collectifs animistes des

14. P. Descola, Par-delà nature et culture, Paris, Gallimard, 2005, p. 263-268.

15. C. LÉVI-STRAuss, La Pensée sauvage, p. 297-298. 
Amériques et de Sibérie, il est pratiqué en Asie du Sud-Est par des collectifs typiques de l'animisme distribué - l'immense majorité des populations montagnardes - dans lesquels le besoin d'un mécanisme permettant de relier des éléments disjoints ne se présente pas avec la même intensité que dans les collectifs analogistes. Il a donc fallu revenir sur la fonction trop exclusive attribuée au sacrifice comme un connecteur nécessaire à l'expression des ontologies analogistes.

On notera d'abord que l'animisme standard, en Asie du Sud-Est et ailleurs, repose sur des formes de relations entre les êtres et de circulation des valeurs assez différentes de celles au sein desquelles le sacrifice se déploie. Dans l'animisme standard, en effet, les humains s'approprient la vie des animaux en les chassant, tandis que les esprits animaux prennent la vie, la chair ou l'âme des humains en contrepartie, que ce retour dans l'échange soit consenti ou pas. Le sacrifice tel qu'il est pratiqué en Asie du Sud-Est évite cette contrepartie désagréable puisque, si les esprits cherchent bien aussi à prendre la vie des humains, ces derniers les trompent en leur donnant à la place la vie des animaux domestiques. Et si, dans l'animisme standard, les animaux se présentent aux humains comme des humains, notamment dans les rêves, parce qu'ils se voient eux-mêmes comme des sujets humains, en revanche les victimes sacrificielles, elles aussi considérées comme des sortes d'humains, ne le sont que parce qu'elles peuvent remplacer les humains vis-à-vis des esprits en raison des propriétés qu'elles partagent avec ces derniers : elles sont intégrées dans un collectif humain où elles témoignent de qualités sociales. Ce remplacement des humains par des animaux pour payer une dette ou défléchir des représailles semble transformer le sacrifice en une opération dans laquelle les relations symétriques entre humains et non-humains caractérisant l'animisme standard sont remplacées par une symétrie pipée : ce ne sont plus les humains qui payent de leur personne le prix des échanges dans lesquels ils s'engagent avec les esprits, mais les animaux qui sont dans leur dépendance. L'examen du sacrifice chez les Rmeet du Laos, un collectif typique de l'animisme distribué, permet toutefois de nuancer cette différence ${ }^{16}$.

Les Rmeet sacrifient des buffles aux esprits domestiques en de nombreuses occasions, notamment en cas de maladie grave, lors des funérailles et des rituels villageois, jadis en hommage aux ancêtres et au cours des mariages. Les Rmeet distinguent trois composantes de la personne : le corps (to), le mouvement de la vie (pääm), et l'âme ( $k l p u)$, celle-ci n'étant présente que dans les humains, les buffles et le riz. L'âme humaine, $k l p u$, est un moyen de communication avec les esprits, en particulier dans les rêves ou elle adopte le point de vue de ces derniers, une plasticité qui se confirme après la mort où elle devient elle-même un esprit. Les buffles sont donc comme les humains par deux éléments de la personne, l'âme et le mouvement de la vie, et ils en diffèrent par un troisième, leur corps, un contraste typiquement animiste. Leur substituabilité aux humains dans le sacrifice vient de ce que les uns et les autres ont la même intériorité : l'âme klpu d'un humain qui lui a été dérobée par un esprit peut être récupérée en échange de l'âme klpu d'un buffle; celle-ci se transforme alors en un buffle pour les esprits des morts. Ajoutons que la maladie n'est pas que l'effet du vol de l'âme, elle résulte aussi de l'intromission d'un esprit dans le corps du patient. Avec le sacrifice, les humains font donc le don aux esprits

16. Voir Guido SPRENGER, «Graded personhood: Human and non-human actors in the Southeast Asian uplands », art. cit., p. 76-84. 
de deux composantes fondamentales de la personne : un corps animal et une âme générique $k l p u$, afin de récupérer ce dont les esprits se sont emparés, à savoir une autre âme générique $k l p u$ et un corps humain.

Le buffle chez les Rmeet semble avoir pour seule fonction d'être un substitut des humains. On ne l'utilise guère comme animal de trait ou pour d'autres fonctions pratiques et il mène une existence très libre, divaguant dans la forêt où il se nourrit par lui-même. Cet animal semi-domestiqué est en même temps vu comme le plus domestique des animaux puisqu'on le place sous la protection de l'esprit habitant la cuisine en lui donnant du sel associé à la fonction protectrice que cet esprit exerce, et en réalisant des rituels pour renforcer le lien du buffle à la maison dont il dépend. En outre, le cosmos des Rmeet est marqué par un perspectivisme accentué. Non seulement le monde des esprits est totalement inversé par rapport à celui des humains, mais les premiers ne font pas les distinctions ontologiques auxquelles les seconds sont attachés. Ainsi, tandis que les esprits des morts voient les humains comme des buffles, les esprits de la forêt voient ces mêmes humains comme des animaux sauvages à chasser, tandis qu'eux-mêmes, les esprits, se voient comme des personnes humaines complètes. Les buffles peuvent ainsi être considérés comme des versions amoindries d'humains, la gradation décroissante des qualités de la personne se poursuivant avec les autres animaux sacrificiels: les cochons sont des buffles diminués, les poulets sont des cochons diminués, etc. Là où l'animisme amazonien et sibérien multiplie les différences de points de vue situés dans des corps qui sont chacun caractéristiques d'une tribu-espèce, l'animisme des Rmeet transpose ce polyperspectivisme à une seule différence majeure, celle qui sépare les humains des esprits, les deux catégories d'êtres qui comptent vraiment et entre lesquelles des relations d'échange biaisées prennent des animaux comme vecteurs. C'est pourquoi le sacrifice ne fonctionne pas ici en mode analogiste, c'est-à-dire comme un connecteur reliant des termes éloignés au moyen d'une série d'identifications; en raison des différences de perspectives entre les humains et les esprits, en effet, les premiers ne peuvent s'identifier aux seconds par l'intermédiaire des animaux. On doit plutôt voir ici le sacrifice comme un classique échange animiste entre humains et non-humains, du type de ceux qui se produisent entre Amérindiens et maîtres du gibier ; mais dans lequel, au lieu de compensations offertes par les humains pour pouvoir tuer des animaux, ceux-ci offrent des substituts d'eux-mêmes pour avoir le droit de n'être pas tués. Ce qui change est la nature des offrandes. Dans l'animisme standard, en Amazonie ou chez les Chewong par exemple, c'est de la nourriture, du tabac, de l'alcool, parfois l'âme des défunts ; ici, ce sont des âmes de substitution, celle des buffles, mais présentées de telle façon que les esprits les confondent avec des âmes humaines. Bref, comme d'habitude avec ce genre de sacrifice, il y a tromperie sur la marchandise.

Mais les Rmeet sont aussi des chasseurs et l'on a donc entrepris de comparer leur logique sacrificielle avec leur rapport au gibier et à l'esprit qui le contrôle. En matière de gros gibier les hommes chassent des cervidés, des cochons sauvages, et jadis des gaurs - des bovidés sauvages. L'esprit qui s'occupe des animaux forestiers s'appelle « Grand-mère la Veuve » et, sans surprise, elle entretient avec eux le même genre de rapport que les Rmeet avec leurs animaux domestiques, ce qui la conduit à relâcher de temps à autre des éléments de son troupeau pour que les humains puissent les chasser. À cela il faut ajouter un esprit de la chasse (Phi Phrean) dont la propitiation rituelle est indispensable aux succès futurs, la cérémonie se déroulant toujours au terme d'une chasse fructueuse ; elle consiste, entre autres opérations, à offrir à cet esprit des morceaux de l'animal abattu. À la différence de ce qui se 
passerait dans l'animisme standard, ce n'est donc pas la maîtresse du gibier qui reçoit une compensation en viande pour l'animal qu'elle a concédé aux humains, mais bien l'esprit de la chasse, le responsable de la bonne fortune du chasseur. Autrement dit, le schème sacrificiel prévaut dans cette opération et non le schème animiste : un animal sauvage appartenant à un chasseur (puisqu'il l'a reçu de la maîtresse du gibier sans compensation) est tué par lui afin d'être consommé de façon cérémonielle en public, une partie de la chair de l'animal étant offerte à un esprit dans l'espoir que celui-ci continuera à favoriser le succès du chasseur. Il faut par ailleurs noter que si le gaur a maintenant disparu de la région occupée par les Rmeet, ce grand bovidé sauvage continue d'être perçu comme un analogue forestier du buffle. Les gaurs sont en effet les seuls animaux sauvages à avoir une âme klpu comme les buffles (et comme les humains). Aussi, lorsqu'un chasseur tuait un gaur et le ramenait au village, le même genre de précautions rituelles s'appliquait que lors du sacrifice du buffle. On fermait le village pour empêcher l'esprit de l'animal de venir s'y venger et il fallait sacrifier des buffles à cet esprit pour apaiser sa colère. En outre, le gaur n'était pas soumis à la juridiction de Grand-mère la Veuve, mais à celle d'un autre esprit, masculin appelé Jao, «seigneur» en lao ; tout comme la maîtresse du gibier ordinaire, le seigneur des gaurs ne demandait rien en échange de ses animaux donnés au chasseur. Toutefois, afin de protéger son troupeau, il pouvait le mener pâturer ailleurs et c'est ainsi que les Rmeet interprètent la disparition de cet animal sur leur territoire. Comme le buffle donc, le gaur présente bien des similitudes avec les humains, et tout comme lui, il doit être compensé auprès des esprits par des dons de vies et d'âmes. On a ainsi une continuité d'échanges rituels ordonnant différents types de dons et de rapports entre humains et non-humains dotés d'une intériorité, mais une continuité dans laquelle ces transactions à première vue typiquement animistes se réorganisent autour d'une logique sacrificielle, c'est-à-dire combinant des mises à mort, différents régimes de substitution et des offrandes. $\mathrm{La}$ victime est toujours un animal appartenant aux humains, qu'il soit domestique ou sauvage, dont la vie ou l'âme est donnée aux esprits, soit en échange d'une vie humaine, soit en compensation d'une vie animale prélevée aux esprits, soit comme une avance pour pouvoir continuer à prélever des vies animales. Là où l'animisme standard joue sur une gamme d'échanges directs entre des partenaires humains et non humains de statut égal, l'animisme distribué des Rmeet et de nombreuses autres populations d'Asie du Sud-Est joue ainsi, par l'intermédiaire du sacrifice, sur une gamme beaucoup plus ample d'échanges de vies, d'intériorités et de physicalités avec des partenaires eux-mêmes plus diversifiés. Cette diversification vient de ce que la relation dyadique de l'animisme entre le chasseur et l'animal hypostasié dans un maître est ici remplacée par l'organisation démultipliée de la circulation sacrificielle - le sacrifiant, le sacrificateur, la victime, le destinataire ; elle vient aussi de la souplesse dans les modes de substitution qu'introduit l'ajout d'une nouvelle catégorie d'êtres, les animaux domestiques, à celles déjà présentes dans l'animisme. Même les moins contrôlés d'entre eux, comme c'est le cas du buffle chez les Rmeet, diffèrent des animaux sauvages qu'apprivoisent les collectifs animistes amazoniens par le fait que ceux-ci, mêmes quand ils vivent dans la maison des humains, continuent d'être sous la juridiction des maîtres du gibier; ils ne sauraient donc être donnés en sacrifice comme des substituts d'humains à ces esprits qui continuent d'en être les maîtres. Les animaux domestiques, par contraste, même élevés en semi-liberté, n'en sont pas moins sous l'autorité des humains, analogues à ces derniers sous certains aspects, et donc substituables à eux dans les échanges. 
Ainsi, donner la vie des animaux domestiques aux métapersonnes dont dépendent l'existence, la santé et la félicité des humains représente pour ces derniers une opération particulièrement avantageuse, à tel point que l'on peut se demander si, dans bien des cas, l'élevage n'est pas apparu pour fournir aux humains des substituts d'eux-mêmes dans le sacrifice, plus que pour tout autre bénéfice pratique.

En conclusion, on a proposé quelques remarques sur le rôle de la domestication animale dans les deux combinaisons ontologiques examinées lors du cours. Tant dans les collectifs animistes d'Asie du Sud-Est que dans le collectif animiste wayuu, les animaux domestiques jouent un rôle central pour déplacer les relations dyadiques et symétriques de l'animisme vers des relations à plusieurs termes caractérisées par l'asymétrie des statuts. Dans les deux cas aussi, il y a une identification des humains aux animaux domestiques qui aboutit, dans le cas wayuu, à ce qu'ils deviennent des substituts de vies humaines dans les rapports intra-humains - compensation matrimoniale et prix du sang - et, dans le cas de l'Asie du Sud-Est, à ce qu'ils deviennent des substituts de vies humaines dans le rapport aux esprits - comme nourriture sacrificielle à la place des humains. Mais les inflexions dans les régimes ontologiques que la relation domesticatoire induit n'ont rien d'automatiques: l'élevage, qui se présente du reste sous des formes très diverses allant du contrôle le plus lâche à la domination la plus étroite, n'est en aucune façon un facteur déclenchant de mutations qui auraient partout des résultats identiques. Ainsi, l'adoption précoce par les Wayuu d'animaux d'élevage européens leur a permis d'actualiser dans des êtres nouveaux un mode d'identification totémique qui existait auparavant à l'état latent, c'est-à-dire purement classificatoire. En postulant une consubstantialité entre les bovins et les matrilignages, les Wayuu ont déplacé l'identification totémique depuis les animaux sauvages éponymes vers les animaux d'élevage ; dans les faits, ils ont « totémisé » le bétail. En Asie du Sud-Est, par contraste, les animaux domestiques ont surtout servi de substituts aux humains pour alimenter les esprits, multipliant ainsi les intermédiaires dans les transactions avec les non-humains, un facteur qui a contribué à diversifier et hiérarchiser le mobilier du monde, et à ouvrir la voie, ce faisant, à une transition graduelle vers une ontologie analogiste pleinement constituée.

\section{SÉMINAIRE}

L'objectif du séminaire était d'offrir un complément au cours en examinant des aspects du thème de l'hybridation des collectifs que le cours n'abordait pas, au premier chef ceux dans lesquels l'ontologie naturaliste intervient. C'est à présent la situation la plus commune. Le phénomène historique de la projection continue sur le reste du monde de la puissance des nations qui ont développé une ontologie naturaliste au cours des derniers siècles a eu pour effet qu'il y a peu de lieux où des éléments idéologiques du naturalisme ne soient pas présents. Il s'agit de ce mouvement historique probablement sans précédent dans l'histoire de l'humanité que l'on appelle la globalisation, dans lequel une manière de concevoir et de pratiquer les rapports entre humains comme les rapports entre humains et nonhumains en y introduisant la médiation de l'échange marchand est en passe de devenir hégémonique, et donc de transformer tous les types de collectifs plus ou moins autonomes, plus ou moins complexes dans leur articulation, que les ethnologues et les historiens avaient pris pour objet d'étude. On n'est donc plus ici face à un problème de logique interne de compatibilité et d'incompatibilité entre des 
ontologies, des cosmologies et des systèmes d'association et de gestion des humains et des non-humains, comme c'est le cas avec les hybridations auxquelles on s'est intéressé dans le cours, mais face à des phénomènes de destruction ou de reconfiguration brutale d'institutions devenues inadaptées au développement du capitalisme marchand et des religions monothéistes et prosélytes qui souvent l'accompagnent. Une façon de faire droit à ces phénomènes était de les aborder dans le séminaire. Aussi a-t-on consacré la plupart des séances à des études de cas du phénomène de la globalisation dans des régions du monde où il s'est produit sous nos yeux dans un laps de temps si bref que l'on peut mieux en maîtriser les étapes et les mécanismes que s'il nous apparaissait dilué sur une très longue durée.

Après une introduction générale par le professeur, des exposés ont permis d'examiner ces questions sur la base d'études circonstanciées. Ont tour à tour été abordés :

- les transformations qui ont affecté au cours des quarante dernières années les Baruya de Nouvelle-Guinée, par Maurice Godelier (directeur d'études EHESS), le $1^{\text {er }}$ février 2018 ;

- la permanence de l'activité rituelle dans la vie civique d'un village nahua au Mexique, par Anath Ariel de Vidas (directeur de recherche CNRS), le 8 février 2018 ;

- le rôle joué par l'argent dans l'organisation des prestations cérémonielles à Dakar, par Ismaël Moya (chargé de recherche CNRS), le 15 février 2018 ;

- les nouvelles formes adoptées par l'imagination prophétique parmi les Amérindiens du Haut Xingu au Brésil, par Emmanuel de Vienne (maître de conférences à l'université Paris-Ouest Nanterre), le 22 février 2018 ;

- l'évolution du paysage pluriethnique à laquelle les Warao du delta de l'Orénoque (Venezuela) doivent s'adapter, par Olivier Allard (maître de conférences EHESS), le $1^{\text {er }}$ mars 2018 ;

- les hybridations ontologiques caractéristiques des populations mélanésiennes de l'île de Makira dans l'archipel des Salomon, par Michael W. Scott (professeur à la London School of Economics), le 8 mars 2018 ;

- le sentiment de la perte culturelle dans le nord du Laos et la réaction patrimoniale qu'elle engendre, par David Berliner (professeur à l'université libre de Bruxelles), le 15 mars 2018 ;

- les attitudes contrastées vis-à-vis de l'esclavage parmi les populations amérindiennes de la côte Pacifique de l'Amérique du Nord, par David Graeber (professeur à la London School of Economics) et David Wengrow (professeur à l'University College de Londres), le 22 mars 2018 ;

- le phénomène de la patrimonialisation du troc dans un marché indigène des Andes argentines, par Olivia Angé (maître de conférences à l'université libre de Bruxelles), le 29 mars 2018.

\section{COLLOQUeS}

\section{Organisation}

Coordination scientifique du colloque de rentrée du Collège de France «Les natures en questions » (avec Alain Fischer, Antoine Georges, Alain Prochiantz, Alain Supiot), qui s'est tenu du 18 au 20 octobre 2017. 


\section{Communications}

- Ph. Descola, «De la Nature universelle aux natures particulières : quelles leçons pour l'analyse des cultures?», colloque international «Les natures en questions », Collège de France, 18-20 octobre 2017.

- Ph. Descola, «Analogism versus animism: discrepancies and resemblances » et «Instituting Amazonian collectives », colloque international « Natures and Cultures in Southeast Asia», organisé par l'Institut de recherche sur l'Asie du Sud-Est contemporaine et le Regional Center for Social Science and Sustainable Development, université de Chiang Mai, Thaïlande, les 7 et 8 novembre 2017.

- Ph. Descola, « ¿Que es la antropología ? » et « Hacia un desarrollo respetuoso de las formas de vida », congrès « Yápankam - Las voces de la investigación en la alta Amazonía Ecuatoriana », Macas (Équateur), 19-21 avril 2018.

- Ph. Descola, «Relationalities», Table-ronde «Thinking about relations in Lowland South America », Institute of Social Anthropology, université d'Oxford, 28 avril 2018.

- Ph. Descola, «Saberes, ontologías, practicas », introduction à la table-ronde «Territorio, agrobio-diversidad y patrimonio biocultural», Universidad de las Américas Puebla, San Andrés Cholula (Mexique), le 14 septembre 2018.

\section{COURS ET CONFÉRENCES À L'EXTÉRIEUR}

- Université Paris-Nanterre, «Comparaison n'est pas raison», conférence à l'invitation de l'Association des étudiants en anthropologie de Nanterre, le 29 septembre 2017.

- Université Chulalongkorn, Bangkok, « Rethinking the Nature/Culture Divide », conférence à l'invitation du département de sciences politiques et de l'Association siamoise de sociologie et d'anthropologie, le 10 novembre 2017.

- Université de Turin, "Ontological pluralism and cultural diversity », leçon inaugurale du corso di laurea en anthropologie culturelle et ethnologie, le 17 janvier 2018.

- Université de Toulouse Jean-Jaurès, « Vers une anthropologie cosmopolitique », le 24 janvier 2018.

- Agence française de développement, Paris, « Politiques de l'anthropocène », le $1^{\text {er }}$ février 2018.

- Institut universitaire européen de Florence, «Structuralism and Ontology: an anthropological journey », le 4 mai 2018.

- Université Stanford, département d'anthropologie, «Cosmopolitics as ontological pluralism », le 16 mai 2018.

- Max-Planck-Institut für Wissenschaftsgeschichte, Berlin, «Is the Anthropocene soluble in ontological pluralism? », le 25 mai 2018.

\section{RECHERCHE}

\section{MISSION ETHNOGRAPHIQUE}

Le professeur s'est rendu en avril 2018 en Équateur, parmi les Shuar et Achuar de la province de Morona Santiago, afin d'étudier les nouvelles formes de revendication 
identitaire suscitées par une inclusion croissante des Amérindiens dans les réseaux mondialisés. La mission était financée par le ministère des Affaires étrangères.

\section{PuBLications}

\section{Ouvrage}

Descola Ph., Cultures, Paris, INSEP/Le Pommier/Carnets Nord, coll. «Homo Ludens», 2017.

\section{Articles}

Descola Ph., «The making of images», in T. Fillitz et P. VAN DER GRIJP (dir.), An Anthropology of Contemporary Art. Practices, Markets and Collectors, Londres/Oxford, Bloomsbury, 2017, p. 25-40.

DesCola Ph., «La boîte aux trésors d'Alain Testart », in D. KARADIMAS, V. LÉCRIVAIN et S. Rostain (dir.), De l'ethnologie à la préhistoire. En hommage à Alain Testart, Paris, L'Herne, «Cahiers d'anthropologie sociale », vol. 16, 2018, p. 9-15.

DESCOLA Ph., «Anthropological comparatisms: generalisation, symmetrisation, bifurcation », in G. LloYd, S. Goldhill et R. GAGNÉ (dir.), Regimes of Comparatism: Frameworks of Comparison in History, Religion and Anthropology, Leyde, Brill, coll. « Jerusalem Studies in Religion and Culture », vol. 24, 2018, p. 401-418. 
